\title{
A spontaneous discharging wound on the abdominal wall
}

\author{
Shmuel Avital, Ron Greenberg, Michal Goldwirth, Nahum Werbin, Yehuda Skornik
}

A 69-year-old woman presented to our out-patient clinic with a 3-year history of a discharging wound from an old scar in the epigastric midline region. She had undergone vagotomy and pyloroplasty 15 years before for a duodenal ulcer. Twelve years later, she noticed a small discharge from an opening in the middle of the old scar. She could not recall any associated pertinent clinical events such as high fever or intense abdominal pain. The discharge would change colour and quantity from time to time, and she finally sought medical assistance because of a marked increase in the amount of discharge.

Physical examination revealed a small opening in the mid-epigastrium and a serotic greenish discharge from it. Blood chemistry values were within normal limits with the exception of a slightly elevated alkaline phosphatase level. Abdominal X-rays were taken shortly after injection of contrast material into the external opening (figure).

Department of Surgery 'A', Tel-Aviv Sourasky Medical Center and the Sackler Faculty of Medicine, Tel-Aviv University, Tel-Aviv, Israel

S Avital

R Greenberg

M Goldwirth

N Werbin

Y Skornik

Accepted 10 March 1998

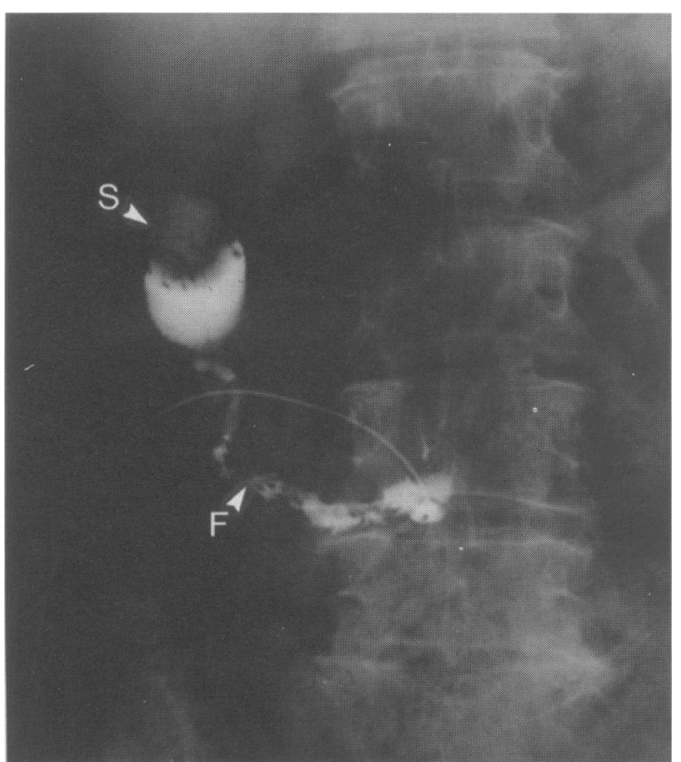

\section{Questions}

1 What are the organ and object seen in this $\mathrm{X}$-ray?

2 What is the diagnosis and what sites are more frequently associated with this kind of pathological communication?
Figure An abdominal X-ray after contrast material injection into the skin opening 


\section{Answers}

QUESTION 1

The organ filled with contrast media is the gallbladder and the enhanced object in it is a very large stone ( $S$ in figure).

QUESTION 2

The diagnosis is cholecystocutaneous fistula which was created spontaneously. The more frequent sites for biliary fistulae are duodenum, colon and stomach.

\section{Outcome}

The patient was taken to surgery. During surgery, a fistulous tract was identified emerging from the fundus of the gallbladder $(F$ in figure), which had adhered to the internal abdominal wall, into the skin. The gallbladder contained a very large stone. A cholecystectomy and excision of the fistulous tract were done. Postoperative recovery was uneventful.

\section{Discussion}

Spontaneous biliary fistulae may be internal or external, most being internal. The internal type communicate to the enteric lumen, mainly to the duodenum and, to a lesser extent, to the colon and stomach $(77 \%, 15 \%$, and $6 \%$, respectively). Other rare types of internal biliary fistulae, such as those that terminate in the urinary system or the bronchial tree, have also been reported. ${ }^{12}$

An external spontaneous biliary fistula erupting to the skin surface is rare. It is defined as a rupture of the gallbladder through all abdominal wall layers with the creation of a fistulous tract to the skin, not preceded by biliary surgery or trauma. The first description of a spontaneous cholecystocutaneous fistula is credited to Thilesus in $1670 .^{2}$ In 1890 , Courvoisier published a large series describing 169 patients with spontaneous external biliary fistulae draining to the abdominal wall. ${ }^{3}$ In 1949, Henry and Orr ${ }^{2}$ reviewed 37 patients with external biliary fistulae who had been diagnosed since 1890 . Since then, only sporadic reports of this biliary tract disease complication have appeared, mainly as case reports of patients with neglected biliary disease. $^{4}$

External biliary fistula formation is mainly due to obstructive gallstone disease and, less frequently, due to a gallbladder adenocarcinoma occluding the cystic duct. ${ }^{5}$ The process of fistulisation is precipitated by obstruction of the cystic duct. When pressure rises in the gall-

1 Glenn F, Reed C, Grafe WR. Biliary enteric fistula. Sur Gynecol Obstet 1981;153:527-31.

2 Henry CL, Orr TG. Spontaneous external biliary fistula. Surgery 1949;26:641-6.

3 Courvoisier L. Pathologie und chirurgie der gallerwege. Leipzig. FCW Vogel, 1890

4 Ruderman RL, Laird W, Reingold MM, Rosen IB. External biliary fistula. Can Med Assoc f 1975;113:875.

\section{Learning points}

- spontaneous external biliary fistulae are rare and usually associated with neglected gallstone disease

- the majority of cases manifest acutely as an abdominal wall abscess connected to the gallbladder; fewer cases will manifest as a chronic discharging wound

- evaluation is done by ultrasound and fistulography

- cholecystectomy and excision of the fistulous tract are the treatment of choice for most patients

- spontaneous closure of the fistulous tract has been reported

bladder, a perforation may ensue, with concomitant adhesions to the internal abdominal wall and the creation of an abdominal wall abscess. Spontaneous or surgical drainage of the abscess can lead to the formation of a fistula. However, this complication may not be preceded by an obvious episode of acute cholecystitis, and the only manifestation may be of chronic discharging sinuses, as in our case and those in other reports. ${ }^{6}$ The majority of the patients with external spontaneous fistulae are elderly women, ${ }^{2}$ reflecting the high incidence of gallstone disease in that population.

Most external fistulae drain to an opening in the right upper abdominal quadrant, and less commonly to the umbilical area. Henry and Orr found that $47 \%$ of fistulae in their series opened to the right upper abdominal quadrant, while $27 \%$ opened to the umbilical region. ${ }^{2}$ Other unusual external sites include the right iliac fossa, right groin, anterior chest wall and back. ${ }^{4}$

Initial treatment consists of drainage and antibiotic administration followed by evaluation with abdominal ultrasound and fistulography. Surgery is invariably indicated and includes cholecystectomy with an excision of the fistulous tract. However, spontaneous closure of the fistula is possible, as recorded in a few patients. $^{2}$ Davis et $a l^{6}$ described a patient treated by direct exploration and extraction of the gallstones through the fistulous tract after which there was a spontaneous closure of the fistula.

\section{Final diagnosis}

Cholecystocutaneous fistula.

Keywords: gallstone disease; biliary fistula; fistulography

5 Hoffman L, Beaton H, Wantz G. Spontaneous cholecystocutaneous fistula: a complication of neglected biliary tract disease. F Am Geriatr Soc 1982;14:632-4.

6 Davies MG, Tadros E, Gaine S, McEntee GP, Gorey TF, Hennessy TP. Combined internal and external biliary fistulae treated by percutaneous cholecystlithotomy. $B r \mathcal{F}$ Surg 1989;76:1258. 